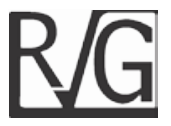

\title{
Responsabilidad social y desempeño financiero en multinacionales: influencia de la diversificación internacional
}

\author{
Aguilera-Caracuel, Javier* \\ Guerrero-Villegas, Jaime ${ }^{* *}$ \\ Morales-Raya, Matilde ${ }^{* \star \star}$
}

\section{Resumen}

La relación entre responsabilidad social corporativa y el resultado financiero de las organizaciones ha sido ampliamente debatida. El objetivo de este trabajo es analizar la relación entre iniciativas de responsabilidad social corporativa y resultado financiero en el contexto de las empresas multinacionales, así como el efecto moderador de la diversificación internacional sobre dicha relación. Para ello fue estimado un modelo estático de datos de panel con efectos fijos sobre una muestra de 113 empresas multinacionales estadounidenses de los sectores químico, energético y de maquinaria industrial. Los resultados muestran que no existe relación directa entre desarrollo de iniciativas socialmente responsables y desempeño financiero. Sin embargo, la presencia de estas empresas en regiones con perfil institucional diferenciado puede conducir a que prácticas de responsabilidad social más visibles por parte de los grupos de interés contribuyan a una mejora sustancial de su rendimiento financiero. Las empresas multinacionales altamente diversificadas pueden adquirir conocimiento valioso y diverso que redunde en una mejora de su desempeño financiero y social.

Palabras clave: empresas multinacionales; responsabilidad social corporativa; resultado financiero; diversificación internacional.

Recibido: 29-07-13 Aceptado: 15-07-15

* Universidad Pablo de Olavide (España), Facultad de Ciencias Empresariales, Departamento de Organización de Empresas y Marketing. E-mail: jagucar@upo.es

** Universidad Pablo de Olavide (España), Facultad de Ciencias Empresariales, Departamento de Organización de Empresas y Marketing. E-mail: jguevil@upo.es

*** Universidad de Granada (España), Facultad de Ciencias Económicas y Empresariales, Departamento de Organización de Empresas. E-mail: matmor@ugr.es 


\title{
Multinational Enterprises' Social Responsibility and Financial Performance: The Influence of International Diversification
}

\begin{abstract}
The relation between corporative social responsibility and the financial outcome of the organizations has been widely debated. The aim of this research is to analyze the relation between corporative social responsibilities initiatives and the financial outcome in the multinational enterprises context, as well as the dampening effect of international diversification on such relation. In order to do so, a static panel data model with fixed effects on a 113 US multinational companies sample from the chemical, energetic and industrial machinery sector was estimated. The results show that the direct relation between socially responsible initiatives development and the financial performance is nonexistent. Nevertheless, the presence of such companies in regions with institutional differentiated profile may lead to more visible social responsibility practices from the interest groups that contribute to a substantial improvement of their financial performance. The highly diversified multinational companies may obtain valuable and diverse knowledge resulting in a financial and social performance improvement.
\end{abstract}

Keywords: multinational enterprises; corporative social responsibility; financial outcome; international diversification.

\section{Introducción}

A lo largo de las últimas décadas se aprecian fuertes presiones por parte de consumidores, proveedores, empleados, inversores, organizaciones no gubernamentales, así como por parte de los poderes públicos para que las empresas inviertan en el desarrollo y puesta en marcha de prácticas de Responsabilidad Social Corporativa (RSC) (McWilliams y Siegel, 2000).

Las empresas más comprometidas con la problemática social se caracterizan por llevar a cabo iniciativas socialmente responsables materializadas en políticas, programas y prácticas, con tienen un efecto positivo en el bienestar de los diferentes grupos de interés (Perrini et al, 2011). Como resultado de todo ello, la organización formula e implanta una serie de estrategias que permiten afianzar las relaciones con los agentes del entorno (Waddock, 2004).

Ante las presiones referidas anteriormente, las organizaciones están respondiendo de diferente forma en cuanto a la adopción de iniciativas en materia social (McWilliams y Siegel, 2000). Algunas empresas han destinado parte de sus recursos al desarrollo de políticas y prácticas socialmente responsables y han fortalecido sus códigos de conducta éticos (Cragg, 2005), incrementando así su transparencia y legitimidad (Bansal, 2005). Sin embargo, directivos de otras empresas se resisten a llevar a cabo tales prácticas argumentando que destinar recursos $y$ esfuerzos a iniciativas que no forman parte de la actividad principal de la organización podría comprometer su buena marcha financiera (Jensen, 2001; Sundaram y Inkpen, 2004). 
La RSC cobra especial relevancia en el caso de las Empresas Multinacionales (EMs), principalmente por dos motivos. En primer lugar, dichas empresas son consideradas como agentes propulsores del desarrollo económico y social a nivel global y local (Kolk y Pinkse, 2008; Kolk y van Tulder, 2010). De hecho, algunas EMs han desarrollado importantes iniciativas en el ámbito de la sanidad pública, de la educación, del empleo, de la protección de derechos humanos (Margolis y Walsh, 2003), así como notorios avances en materia medioambiental (Hart, 1995; Marcus y Fremeth, 2009).

En segundo lugar, las EMs se caracterizan por tener diferentes unidades organizativas, matrices y subsidiarias, ubicadas en países con perfiles institucionales diferenciados no sólo en cuanto a los aspectos regulatorios, sino también en relación a los aspectos cognitivos y normativos (Kostova et al, 2008). Como consecuencia de ello, las EMs toman decisiones sobre la adaptación de sus prácticas de gestión, incluidas las vinculadas con las iniciativas de RSC, o si, por el contrario, homogeneizan esas prácticas a lo largo de su entramado organizativo mediante el fortalecimiento de estándares internos (Christmann, 2004; Kostova y Roth, 2002).

Existe un polémico debateen cuanto a la relación entre la RSC y el desempeño financiero de las organizaciones (Margolis y Walsch, 2003; Orlitzky et al, 2003; Perrini et al, 2011; Vogel, 2005). Desde el punto de vista de la teoría de los stakeholders (Freeman, 1984), la relación entre RSC y desempeño financiero es positiva (Choi y Wang, 2009; Margolis y Walsch, 2001; Waddock y Graves, 1997). Por el contrario, desde la óptica de la teoría neoclásica (Friedman, 1970), esta relación es negativa (Barnett, 2007; Jensen, 2001, Scherer y Palazzo, 2011).
Adicionalmente, en otros estudios sobre la relación entre RSC y resultado financiero se ha mostrado inexistente (neutral) debido fundamentalmente a una serie de recursos intangibles condicionantes de dicha relación (Ullman, 1985; McWilliams y Siegel, 2001; Yang et al, 2010). Entre los condicionantes se incluyen las características del entorno (Aragón-Correa y Sharma, 2003), la capacidad de innovación de la empresa o las características del sector de actividad (Hull y Rothenberg, 2008; Russo y Fouts, 1997). Es de hacer notar que en el estudio de la relación entre RSC y desempeño financiero, se ha prestado escasa atención al efecto moderador de la diversificación internacional a la hora de rentabilizar las iniciativas sociales llevadas a cabo por la empresa.

La diversificación internacional se define como el número de mercados donde la empresa actúa y la importancia otorgada a cada uno de estos mercados (Hitt et al, 1997:767). Este concepto es de especial importancia en el caso de las EMs porque éstas se caracterizan por operar y tener presencia física en países con perfil institucional diferenciado (Durán-Herrera y Bajo-Davó, 2013; Kostova y Roth, 2002). Strike et al (2006) estudiaron el efecto de la diversificación internacional de las EMs en sus prácticas de RSC. Concretamente, mostraron que la diversificación internacional conducía al desarrollo de prácticas social-mente responsables e irresponsables simultáneamente.

Por un lado, la diversificación internacional permite a las EMs conocer las características de cada mercado, pudiendo trasladar de manera efectiva sus iniciativas sociales a distintos grupos de interés, gestionar las diferencias regulatorias existentes e incluso fortalecer sus propios estándares sociales y medioambientales (Strike et al, 2006). Por otro lado, una alta 
Responsabilidad social y desempeño financiero en multinacionales...

Aguilera-Caracuel, Javier; Guerrero-Villegas, Jaime y Morales-Raya, Matilde

diversificación internacional puede elevar los costos de coordinación y ralentizar la asimilación de nuevo conocimiento debido a los complejos mecanismos de control empleados entre matriz y subsidiarias (Hitt et al, 1997 y 2006). Esto último, puede llevar a las EMs, o algunas de sus unidades, a prescindir del desarrollo de iniciativas socialmente responsables y actuar de manera oportunista con el objetivo de alcanzar un beneficio financiero en el corto plazo.

El objetivo de esta investigación es analizar la relación entre iniciativas de responsabilidad social corporativa y el resultado financiero en el contexto de las EMs, así como el efecto moderador de la diversificación internacional sobre dicha relación. Con base en la teoría del conocimiento (Eriksson et al, 2000; Chetty et al, 2006), en este trabajo se argumenta que a través de la diversificación internacional las EMs adquieren conocimiento valioso y diverso procedente de los diferentes mercados donde operan. Este conocimiento permite a las EMs instaurar de forma efectiva iniciativas de carácter social que benefician a la sociedad, y al mismo tiempo ser rentables desde el punto de vista financiero para las EMs.

A fin de analizar estas relaciones, la muestra utilizada corresponde a 113 EMs estadounidenses con datos longitudinales para el período 2005-2010. La elección de Estados Unidos como país donde se ubica la matriz se debe principalmente a que las EMs con sede en dicho país tienen una relevancia notoria e impacto socio-económico no sólo en su país de origen, sino en otros mercados similares o diferentes desde un punto de vista institucional.

Partiendo de la información disponible en Standard \& Poors' (Capital $I Q)$, se llevó a cabo un muestreo aleatorio simple lo cual permitió seleccionar un total de 100 EMs del sector químico, 100 EMs del sector energético y otras 100 EMs del sector de maquinaria industrial. La información financiera se obtuvo de la base de datos Standard \& Poors' (Capital $I Q)$, mientras que la información referida a RSC proviene de la base de datos Kinder, Lydenberg, Domini (KLD). Tras considerar la información disponible de RSC y financiera para el total de empresas y periodo de estudio, la muestra final comprende un total de 113 EMs y 672 observaciones.

La distribución de la muestra por sectores es la siguiente: $49 \mathrm{EMs}$ pertenecen al sector de maquinaria industrial (289 observaciones), $45 \mathrm{EMs}$ al sector químico (270 observaciones) y 19 EMs al sector energético (113 observaciones). Estos tres sectores de actividad se caracterizan por tener un gran impacto medioambiental y una repercusión importante en el ámbito social (King y Shaver, 2001).

El modelo de efectos fijos fue estimado mediante técnicas aplicables a datos de panel, utilizando el programa estadístico STATA. Para determinar si procedía aplicar efectos fijos o aleatorios en el modelo se aplicó el contraste de Hausman (Hausman, 1978). Estos análisis permiten aportar datos empíricos que arrojan luz al debate académico sobre a) qué efecto tiene la RSC en el desempeño financiero en el contexto de las EMs, y b) cuál es el rol de la diversificación internacional en dicha relación.

\section{Teoría del conocimiento en empresas internacionales}

La teoría del conocimiento parte de la idea de que el conocimiento es uno de los recursos más importantes y relevantes para las empresas (Barney, 1991; Nonaka, 1994). Estudios previos han analizado 
cómo el proceso de internacionalización de las empresas contribuye a la generación de conocimiento valioso que es fuente de ventaja competitiva (Blomstermo et al, 2004; Petersen et al, 2008). De hecho, para Nachum y Zaheer (2005) la decisión de expansión internacional de sus actividades no sólo viene motivada por su desempeño financiero, sino también por un acceso privilegiado a conocimiento y otros recursos.

En este sentido, la reputación y la imagen pública de las empresas son recursos intangibles que permiten alcanzar ventaja competitiva (Barney 1991; Deephouse, 2000). Las iniciativas socialmente responsables llevadas a cabo por las EMs pueden contribuir a mejorar dichos recursos intangibles y ayudar a mejorar el desempeño de las EMs.

Las EMs son más visibles y están sujetas a un mayor escrutinio por parte de los grupos de interés. Por consiguiente, suelen prestar una mayor atención a su reputación e imagen pública (Fombrun, 1996). Diversos estudios sugieren que las presiones globales e institucionales han empujado a las EMs a aumentar sus iniciativas socialmente responsables (Sharfman et al, 2004). Entre estas iniciativas se encuentra el establecimiento de estándares en materia laboral, respecto al pago, mano de obra infantil, con el fin de proteger la imagen pública de las EMs (Caves, 1996).

El conocimiento proveniente de mercados foráneos se acumula de forma gradual y paulatina a lo largo del entramado organizativo de la empresa (Eriksson et al, 2000; Johanson y Valhne, 1977). Por tanto, las empresas necesitan un periodo de tiempo para poder adquirir, asimilar y desarrollar conocimiento nuevo procedente del extranjero (Chetty et al, 2006). Por otro lado, para las empresas nacidas globales ('born-global firms') el conocimiento juega un papel clave para expandir sus operaciones rápidamente por otros mercados y diferenciarse de las empresas locales (Andersson y Wictor, 2003; Autio et al, 2000; Zahra, 2005).

\section{Responsabilidad social corporativa y resultado financiero}

Los resultados relativos a la relación existente entre prácticas de RSC y desempeño financiero son controvertidos y no concluyentes (Barnett, 2007; Vogel, 2005). Todo ello viene motivado, especialmente, por la forma de medir la RSC de las organizaciones (Margolis y Walsh, 2003) así como por la posible relación de causalidad entre RSC y resultado financiero (Orlitzky et al, 2003). Existen básicamente tres posturas en relación a este debate académico:

En primer lugar, los estudios agrupados en la corriente denominada "hipótesis de las variables moderadoras" (Gómez-García, 2008) argumentan la inexistencia de relación entre RSC y desempeño financiero debido a la presencia de una serie de recursos intangibles condicionantes que condicionan esta relación (McWilliams y Siegel, 2001; Yang et al, 2010).

Características del entorno tales como la incertidumbre, la complejidad y la munificencia condicionan la efectividad y rentabilidad de las prácticas de RSC (Aragón-Correa y Sharma, 2003). La innovación y el nivel de diferenciación en un sector de actividad también ejercen un efecto moderador en la relación entre RSC y mejora del desempeño financiero (Hull y Rothenberg, 2008). Esta relación positiva es más evidente en empresas con bajos niveles de innovación y con bajos niveles de diferenciación en el sector ya que, en 
Responsabilidad social y desempeño financiero en multinacionales...

Aguilera-Caracuel, Javier; Guerrero-Villegas, Jaime y Morales-Raya, Matilde

tales casos, las prácticas de RSC actúan como fuente de diferenciación.

Dentro de este primer grupo, también se encuentran trabajos que resaltan la necesidad de la existencia de determinados recursos intangibles para que las iniciativas de RSC redunden en un mejor desempeño financiero. En este sentido, dicha relación se ve reforzada ante la existencia de una mayor reputación de la empresa (Strong et al, 2001), una gestión apropiada de los recursos humanos (Russo y Harrison, 2005) y una cultura organizativa que promueva las iniciativas socialmente responsables (HowardGrenville y Hoffman, 2003).

En segundo lugar, otros estudios argumentan una relación negativa entre las prácticas de RSC y el desempeño financiero. Desde la perspectiva de los postulados neoclásicos (Friedman, 1970), se defiende que la inversión en recursos destinados a la puesta en marcha de iniciativas vinculadas a la RSC generan un exceso de costos poniendo así en peligro el mantenimiento de la ventaja competitiva de las organizaciones (Barnett, 2007; Scherer y Palazzo, 2011).

Concretamente, los recursos destinados a programas y acciones sociales revierten de forma negativa en la eficiencia operativa así como en el reparto de dividendos a los inversores. Junto a ello, la diversificación en cuanto a actividades y programas sociales por parte de la empresa podría descentrar a la empresa de la actividad esencial para las que fue creada (Jensen, 2001).

Finalmente, numerosos estudios sostienen una relación positiva entre RSC y desempeño financiero debido fundamentalmente a la capacidad de la propia empresa para poder responder a las demandas de los grupos de interés en los diferentes contextos donde operan (Margolis y Walch, 2003; Waddock y
Graves, 1997). En este sentido, según la teoría de los stakeholders (Freeman, 1984), el hecho de poder hacer frente a las necesidades y demandas impuestas por los distintos grupos de interés permite a las organizaciones disminuir sus costos de transacción, aumentar su legitimidad y su confianza, y obtener ventaja competitiva en relación a sus competidores (Choi y Wang, 2009).

Por el contrario, las empresas que actúan de forma socialmente irresponsable con el objetivo de disminuir sus costos implícitos (por ejemplo, localizando actividades de alto impacto medioambiental en países con menores exigencias legales), incurrirán posteriormente en altos costos explícitos derivados de una falta de legitimidad, sufriendo el rechazo social de los grupos de interés (Bansal, 2005).

Siguiendo con la teoría de los stakeholders (Freeman, 1984), la RSC tiene un impacto positivo en la captación y productividad de los trabajadores. La puesta en marcha de iniciativas en el ámbito social fomenta la creación de un buen ambiente de trabajo. Esto se traduce en un mayor compromiso del trabajador con la organización, mayor productividad de los empleados $y$, en definitiva, en un mejor desempeño financiero para la empresa (Tencati y Zsolnai, 2009).

Las empresas más comprometidas con la problemática social tienen también más facilidad para contratar a los "mejores trabajadores" del mercado y contar con sus iniciativas proactivas para la puesta en marcha de nuevas prácticas de RSC efectivas, así como con los programas de formación necesarios para llevar a cabo esas iniciativas (Ramus y Steger, 2000). Las iniciativas sociales de la compañía también pueden mejorar la satisfacción y lealtad de los clientes (Lichtenstein et 
al, 2004), y las colaboraciones con entes públicos y privados en materia social (Post et al, 2002).

En relación al aspecto medioambiental, la incorporación de avances en los procesos productivos de la empresa no sólo puede minimizar el impacto nocivo sobre el medio ambiente, sino también reducir costos operativos (por ejemplo, costos de materias primas) y aumentar la productividad de los trabajadores (Hoffman, 2000; Porter y van der Linde, 1995). Además, la mejora de la imagen de la empresa puede atraer a nuevos inversores (Bauer et al, 2007; Sharfman y Fernando, 2008).

En el caso de las EMs, la puesta en marcha de políticas y prácticas de RSC resulta especialmente relevante. Primero, dichas empresas pueden beneficiarse de la reducción de sus costos operativos mediante la creación de estándares sociales y medioambientales sólidos a lo largo de su entramado organizativo (Christmann, 2004). Como consecuencia de ello, van a poder reforzar su coherencia interna (Kostova et al, 2008).

Segundo, las EMs pueden aumentar su volumen de negocio mediante una mejora de su reputación y legitimidad en los diferentes mercados donde actúan, creándose así una mayor lealtad por parte de clientes e instituciones (Bansal, 2005). En definitiva, la implantación de iniciativas socialmente responsables debería permitir a las EMs reforzar su coherencia interna, mejorar su reputación y la lealtad de sus clientes lo que redunda en una mejora del desempeño financiero. Por tanto, se plantea la Hipótesis 1.

Hipótesis 1: Las iniciativas en materia de RSC en las EMs están relacionadas positivamente con su desempeño financiero.

\section{Efecto moderador de la diversificación internacional de EMs en la relación RSC - desempeño financiero}

La diversificación internacional de las EMs puede acarrear costos elevados de coordinación pues se trata de organizaciones muy complejas: por un lado, manejan un gran volumen de información y por otro cuentan con diferentes unidades ubicadas en entornos con peculiaridades institucionales (Kostova y Roth, 2002; Kostova et al, 2008; Peng et al, 2008). Interactuar con diferentes culturas, niveles de desarrollo económico diferenciados, así como satisfacer necesidades diversas de clientes y resto de grupos de interés (Van Raaij, 1997), dificulta a las EMs a centralizar y estandarizar sus prácticas de gestión (incluyendo las políticas y prácticas de RSC).

El uso de sistemas descentralizados de gestión, puede provocar la existencia de duplicidades innecesarias en las distintas unidades organizativas y problemas a la hora de transferir recursos y capacidades a lo largo del entramado organizativo (Sambharya, 1995). Por otro lado, pueden existir presiones financieras de los inversores que frenen a las organizaciones a invertir en avances en materia social (Strike et al, 2006).

A pesar de la existencia de los estudios anteriormente indicados, numerosos trabajos defienden el papel fundamental de la diversificación internacional en las EMs, a la hora de aprovechar las oportunidades que proporcionan los mercados foráneos (Barkema y Vermeulen, 1998; Rugman, 1981). La actuación de las empresas en diferentes contextos les permite aprovechar ventajas derivadas de localización, 
Responsabilidad social y desempeño financiero en multinacionales... Aguilera-Caracuel, Javier; Guerrero-Villegas, Jaime y Morales-Raya, Matilde

aprendizaje y adquisición de conocimiento (Thomas y Eden, 2004).

Concretamente, la diversificación internacional permite a las empresas tener acceso a recursos tangibles e intangibles necesarios para el desarrollo de capacidades innovadoras (Bartlett y Ghoshal, 1989; Kotabe, 1990). La empresa puede desarrollar capacidades innovadoras a través de la adquisición de nuevo conocimiento de los mercados con perfil cultural e institucional diferenciado en los que opera (Kostova et al, 2008). Otro de los beneficios importantes de la diversificación internacional se relaciona con la reducción del riesgo derivada de la actuación en múltiples mercados (Hitt et al, 1997). Como consecuencia de todo ello, en las últimas décadas existe una tendencia clara y creciente a que las organizaciones aumenten su presencia internacional (Casillas et al, 2010).

En el contexto de las EMs, Hymer (1976) y Rugman (1981) sostienen que la diversificación internacional permite a dichas organizaciones aprovechar las ventajas provenientes de los diferentes mercados mediante una adecuada transferencia de conocimiento valioso a lo largo de todas las matrices y subsidiarias (Kostova y Roth, 2002; Aguilera-Caracuel et al, 2012). Todo ello permite a dichas empresas el uso eficiente de economías de escala y aprendizaje (Kogut, 1985).

Con relación a la problemática social, numerosos estudios defienden como las presiones globales $e$ institucionales de cada mercado contribuyen a que las EMs alcancen niveles elevados de RSC mediante la creación de estándares relacionados con la seguridad en las condiciones laborales, protección de minorías sociales, promoción del empleo y riqueza o preservación del entorno natural (Kolk y van Tulder, 2010). Son muchos los trabajos que han prestado especial interés a la problemática medioambiental de las EMs, centrándose en aspectos tales como regulaciones del país de origen y de destino (Rugman y Verbeke, 1998) o la influencia de las presiones de los diferentes grupos de interés en las iniciativas medioambientales de dichas organizaciones (Bansal, 2005; Christmann, 2004).

Strike et al (2006) afirman que las EMs con alto nivel de diversificación internacional van a poder comunicarse de manera efectiva con los diferentes grupos de interés, captar las necesidades y expectativas de los mismos, tener un papel activo en el diseño de regulaciones nacionales e internacionales en el ámbito social y medioambiental, y, por último, crear estándares sociales y medioambientales propios que superen las limitaciones legales impuestas en cada mercado. Dichas empresas son capaces de mantener relaciones estables y basadas en la confianza con gobiernos y poderes públicos que les permiten tener acceso privilegiado a subvenciones y permisos para actuar en diferentes mercados (Luo, 2001).

Numerosos trabajos han tenido en cuenta la relación existente entre diversificación internacional y RSC (Strike et al, 2006), así como la relación entre desempeño social y financiero de la empresa (Perrini et al, 2011). Sin embargo, escasa atención se ha prestado a cómo la diversificación internacional de las EMs puede condicionar la forma en la que los recursos destinados a prácticas de RSC pueden revertir en una mejora sustancial en su desempeño financiero. Una alta diversificación internacional de las EMs puede favorecer estar en contacto con diferentes grupos de interés y captar las necesidades de diferentes entornos diversos.

Esa presencia activa puede permitir a las EMs llevar a cabo una 
serie de iniciativas sociales efectivas que redunden positivamente en su resultado financiero gracias a un aumento de su cifra de negocios, motivado por la mejora sustancial en su reputación y legitimidad (Bansal, 2005; Kostova et al, 2008). La mejora del desempeño financiero puede también deberse a una optimización de recursos y procesos, así como a la creación de estándares sociales y medioambiental que permiten fortalecer la estructura organizativa de la empresa (Bartlett y Ghoshal, 1989). Por todo ello, se propone la Hipótesis 2.

Hipótesis 2: La diversificación internacional de las EMs ejerce un efecto moderador positivo en la relación existente entre RSCresultado financiero.

\section{Variables y resultados del análisis}

El modelo a estimar considera como variable dependiente el desempeño financiero y como variables independientes las prácticas de RSC y la diversificación internacional, así como algunas variables de control. La diversificación internacional viene determinada por el número de países y/o regiones donde la empresa desarrolla sus actividades (Barkema y Vermeulen, 1998; Tallman y Li, 1996). Para medir el grado en el que las EMs operan en mercados institucionalmente diferenciados se utilizó un Índice de Entropía Regional (IEG) (Hitt et al, 1997). EI IEG se define de la siguiente forma:

$$
I E G=\sum_{i=1}^{n} P_{i} \cdot \operatorname{Ln}\left(\frac{1}{P_{i}}\right)
$$

donde $P_{i}$ se refiere al porcentaje de ventas destinado a cada región y $L n$ (1/ $P$ ) representa el peso otorgado a cada región (logaritmo neperiano de las ventas de la región i). Los países se agruparon en seis regiones diferenciadas en cuanto a su perfil institucional y cultural (Yeoh, 2004): Latinoamérica, Asia, América del Norte (incluye México y Canadá), Australia, Europa Occidental y Europa del Este.

Las prácticas de RSC se obtuvieron de la base de datos KLD. KLD valora a las empresas en una serie de dimensiones de RSC. Este estudio considera aquellas dimensiones sociales que juegan un papel esencial a la hora de establecer relaciones con los diferentes grupos de interés y que contribuyen significativamente en la formulación y en la implantación de la estrategia corporativa de las empresas (Prahalad y Hamel, 1994).

En este estudio se utilizaron los siguientes indicadores: relaciones con la comunidad local, relaciones con mujeres y grupos desfavorecidos, relaciones con empleados, impacto en el entorno natural (medio ambiente) y características socialmente res-ponsables de los productos ofertados por las organizaciones (Waddock y Graves, 1997). Cada empresa valorada por KLD tiene una puntuación para cada indicador de RSC que oscila entre +2 y -2 puntos, siendo +2 una fortaleza, 0 una posición neutral y, -2 una debilidad.

Tras realizar un análisis factorial exploratorio de los cinco indicadores usando el método de componentes principales (Hair et al, 2009) y con rotación VARIMAX, se distinguieron dos factores (Tabla 1). Cada factor en su conjunto tiene autovalores mayores a 1 , el alpha de Cronbach excede en ambos factores el valor de 0.7 (Nunnally y Bernstein, 1995) y las cargas factoriales de los indicadores de cada factor exceden el 
Responsabilidad social y desempeño financiero en multinacionales...

Aguilera-Caracuel, Javier; Guerrero-Villegas, Jaime y Morales-Raya, Matilde

valor de 0.5. En base a este análisis se distinguieron dos grupos de prácticas de RSC. Las prácticas de "comportamientos socialmente responsables con los grupos de interés" (Factor 1) menos visibles por los stakeholders y las prácticas de "comportamiento medioambiental e impacto de los productos" (Factor 2) más fácilmente visibles por los stakeholders.

\section{Tabla 1}

\section{Análisis Factorial Exploratorio de las Políticas y Prácticas de RSC}

\begin{tabular}{|c|c|c|c|c|}
\hline & $\begin{array}{l}\text { Comportamientos } \\
\text { socialmente } \\
\text { responsables con los } \\
\text { diferentes grupos de } \\
\text { interés (Factor 1) }\end{array}$ & $\begin{array}{l}\text { Cargas } \\
\text { factoriales }\end{array}$ & $\begin{array}{c}\text { Comportamiento } \\
\text { medioambiental } \\
\text { e impacto de los } \\
\text { productos (Factor 2) }\end{array}$ & $\begin{array}{c}\text { Cargas } \\
\text { Factoriales }\end{array}$ \\
\hline & $\begin{array}{l}\text { Relaciones con la } \\
\text { comunidad local }\end{array}$ & 0.73 & & \\
\hline & $\begin{array}{l}\text { Relaciones con mujeres y } \\
\text { grupos desfavorecidos }\end{array}$ & 0.73 & & \\
\hline & Relaciones con empleados & 0.60 & & \\
\hline & & & $\begin{array}{c}\text { Impacto en el medio } \\
\text { ambiente }\end{array}$ & 0.77 \\
\hline & & & $\begin{array}{l}\text { Caracteristicas } \\
\text { socialmente } \\
\text { responsables de los } \\
\text { productos ofertados }\end{array}$ & 0.66 \\
\hline Autovalores & 1.54 & & 1.17 & \\
\hline$\%$ varianza & 0.45 & & 0.33 & \\
\hline $\begin{array}{l}\% \text { acumulado } \\
\text { varianza }\end{array}$ & 0.45 & & 0.78 & \\
\hline $\begin{array}{l}\text { Alpha de } \\
\text { Cronbach }\end{array}$ & 0.70 & & 0.72 & \\
\hline
\end{tabular}

Método de estimación: Método de componentes principales. Rotación VARIMAX.

Fuente: Elaboración propia, a partir de dimensiones de RSC incluidas en la base datos KLD.

Para medir el desempeño financiero se utilizó la ratio de Rentabilidad sobre los Activos (ROA) (Chih et al, 2010; Razafindrambinina y Sabran, 2014). Dicho ratio permite evaluar la eficiencia de la empresa conseguida con su volumen de activos. Para controlar el tamaño se utilizó el volumen de ingresos ("total revenues") de cada empresa multinacional en su conjunto (se incluyen todas las unidades organizativas de la empresa multinacional)
(Karim et al, 2013; Kaufmann y Körte, 2010).

El efecto de la industria se controló incorporando dos variables dicotómicas para dos de los tres sectores de actividad (Christmann y Taylor, 2001): sector químico y sector de maquinaria industrial. El grado de internacionalización, es decir, la presencia de la empresa en mercados extranjeros se midió a través del ratio del total de ventas procedente de los mercados 
extranjeros entre el total de ventas de la empresa multinacional en su conjunto (Hitt et al, 1997). Para observar la relación entre el desempeño social y el desempeño financiero y el efecto moderador del grado de internacionalización se ha utilizado una regresión moderadora con datos de panel $\mathrm{y}$ efectos fijos. El modelo utilizado ha sido el siguiente:

$R O A=\alpha_{0}+\alpha_{1}$ Comportamientos sociales con los grupos de interés (Factor 1) $)_{\text {it }}+\alpha_{2}$ Comportamiento medioambiental e impacto de los productos (Factor 2) it $+\alpha_{3}$ Sector maquinaria industrial ${ }_{i t}+\alpha_{4}$ Sector químico $_{i t}+\alpha_{5}$ Volumen de ingresos $_{\text {it }}$ $+\alpha_{6}$ Porcentaje de ventas en el extranjero $_{i t}+\alpha_{7}$ Diversificación internacional $+\alpha_{8}$ Factor $1_{i t} x$
Diversificación internacional $_{\text {it }}+$ $\alpha_{9}$ Factor $2_{i t} \quad x \quad$ Diversificación internacional $_{i t}+\varepsilon_{i t}$

En la Tabla 2 se detallan los estadísticos descriptivos y la matriz de correlaciones de todas las variables utilizadas en el análisis. De la Tabla 2 cabe destacar que la rentabilidad financiera de las empresas de la muestra representa un valor medio de 0,06. El volumen de ingresos medio es 21,45 y presenta una elevada dispersión. El porcentaje de ventas en el extranjero por término medio es de un $9,96 \%$ La diversificación internacional media es 0,6 . En cuanto a las prácticas de RSC, el valor medio de los comportamientos sociales con los grupos de interés (Factor 1) es de 0,11 y de $-0,18$ para el comportamiento medioambiental e impacto de los productos (Factor 2).

\section{Tabla 2}

\section{Resultados de la Regresión con Estimadores Fijos Relación RSC - Desempeño Financiero}

\begin{tabular}{|c|c|c|c|c|c|c|c|c|c|}
\hline & Media & $\begin{array}{c}\text { Des- } \\
\text { viación } \\
\text { Típica }\end{array}$ & 1 & 2 & 3 & 4 & 5 & 6 & 7 \\
\hline 1, Rentabilidad financiera ( $R O A)$ & 0,06 & 0,04 & 1 & & & & & & \\
\hline 2, Sector maquinaria industrial & 0,43 & 0,50 & $-0,06$ & 1 & & & & & \\
\hline 3, Sector químico & 0,41 & 0,49 & 0,05 & $-0,74^{\prime * *}$ & 1 & & & & \\
\hline 4 , Volumen de ingresos & 21,45 & 45,34 & $0,09^{*}$ & $-0,04$ & $-0,01$ & 1 & & & \\
\hline $\begin{array}{l}5 \text {, Porcentaje de ventas en el } \\
\text { extranjero }\end{array}$ & 9,96 & 25,69 & $0,13^{*+*+1}$ & $-0,06$ & $-0,03$ & $0,09^{*}$ & 1 & & \\
\hline 6, Diversificación internacional & 0,6 & 0,43 & $0,26^{* *+*}$ & 0,03 & $-0,07$ & $0,08^{*}$ & $0,18^{* * *}$ & 1 & \\
\hline $\begin{array}{l}\text { 7, Comportamientos sociales con los } \\
\text { grupos de interés (Factor } 1 \text { ) }\end{array}$ & 0,11 & 0,5 & 0,04 & $-0,06$ & 0,06 & $-0,05$ & $-0,06$ & $-0,03$ & 1 \\
\hline $\begin{array}{l}\text { 8, Comportamiento medioambiental e } \\
\text { impacto de los productos (Factor } 2 \text { ) }\end{array}$ & $-0,18$ & 0,58 & $-0,37$ & $-0,09^{*}$ & $-0,03$ & $-0,1^{*}$ & $-0,08^{*}$ & $-0,01$ & $0,23^{*}$ \\
\hline
\end{tabular}

Número de observaciones $(N)=672$; Número de grupos $=113 ; \dagger p<0.10 ;{ }^{*} p<0.055 ;{ }^{* *} p<0.01$; **t $p<0.001$

Fuente: Elaboración propia, a partir de datos incluidos en KLD y Standard \& Poors' (Capital IQ). 
Responsabilidad social y desempeño financiero en multinacionales...

Aguilera-Caracuel, Javier; Guerrero-Villegas, Jaime y Morales-Raya, Matilde

La Tabla 3 muestra los resultados de la regresión con estimadores fijos. Los Factores de Inflación de la Varianza (FIV) por debajo de 5 muestran que no existen problemas de multicolinealidad en las variables utilizadas (Hair et al, 2009). Las variables han sido estandarizadas con el fin de poder introducir los términos multiplicativos (efectos moderadores) en el modelo (Venkatraman, 1989). Los valores relativos al $R^{2}$ within (0.18) y al estadístico $F$ muestran que existe un buen ajuste en el modelo.

Tabla 3

Resultados de la Regresión con Estimadores Fijos Relación RSC - Desempeño Financiero

\begin{tabular}{lc} 
Constante & -1.49 \\
Sector maquinaria industrial & $(1.53)$ \\
& 25.10 \\
Sector químico & $(20.94)$ \\
& 49.54 \\
Volumen de ingresos & $(46.81)$ \\
& -0.03 \\
Porcentaje de ventas en el extranjero & $(0.15)$ \\
& $0.14^{* *}$ \\
Diversificación internacional & $(0.52)$ \\
Factor 1 (comportamientos sociales & $0.27^{* * *}$ \\
con los grupos de interés) & $(0.37)$ \\
Factor 2 (comportamiento medioambiental & $\mathbf{0 . 0 3}$ \\
e impacto de los productos) & $\mathbf{( 0 . 2 2 )}$ \\
Diversificación internacional x Factor $\mathbf{1}$ & $\mathbf{0 . 0 1}$ \\
& $\mathbf{( 0 . 0 2 )}$ \\
Diversificación internacional x Factor 2 & $\mathbf{0 . 0 2}$ \\
F & $\mathbf{( 0 . 0 3 )}$ \\
$R^{2}$ within & $\mathbf{0 . 1 2}$ \\
Hausman & $\mathbf{( 0 . 0 3 )}$ \\
Número de observaciones & $11.21^{* * * *}$ \\
\hline & 0.18 \\
& $20.82^{* * *}$ \\
& 672 \\
& 113 \\
\hline
\end{tabular}

Variable dependiente: Rentabilidad financiera (ROA)

La tabla recoge los coeficientes (estimadores) del modelo de regresión.

Entre paréntesis se recogen las desviaciones típicas.

† $p<0.10 ;{ }^{*} p<0.055 ;{ }^{* *} p<0.01 ;{ }^{* * *} p<0.001$

Fuente: Elaboración propia. 
Con relación a las variables de control, se puede observar como el grado de internacionalización (porcentaje de ventas en el extranjero) influye positivamente en la mejora del desempeño financiero de las EMs. Esta misma tendencia ocurre en el caso de un alto grado de diversificación. Por tanto, estos resultados son consistentes con los trabajos que muestran la existencia de una relación directa y positiva entre internacionalización y resultado financiero (Grant, 1987; Han et al, 1998).

En segundo lugar, los resultados muestran que no existe relación directa significativa entre prácticas de RSC y resultado financiero. La Hipótesis 1 no es respaldada en la muestra. Por tanto, no es posible afirmar que, en todo caso, la puesta en marcha de iniciativas socialmente responsables por parte de las EMs se traduce en un mejor o peor resultado financiero. Este resultado es consistente con estudios previos, los mismos sostienen que la relación entre RSC y desempeño financiero está condicionada por factores intangibles (Aragón-Correa y Sharma, 2003; Hull y Rothenberg, 2008; Yang et al, 2001).

En el caso de las EMs, en este trabajo la diversificación internacional es uno de estos factores que intervienen dicha relación. Las EMs tienen la capacidad de adquirir conocimiento valioso gracias a la interacción en diferentes mercados con un perfil institucional distinto (Kostova et al, 2008) y se relacionan con diversos agentes. Todo ello les permite captar las necesidades que tienen los diferentes grupos de interés en cuanto a la problemática social, y resultando sumamente útil para poner en práctica iniciativas de RSC efectivas y valoradas por los stakeholders de los diferentes mercados donde actúan.

En tercer lugar, se observa que la diversificación internacional de las EMs no ejerce ningún efecto moderador en la relación entre las iniciativas de RSC englobadas en el Factor 1 y el desempeño financiero. Dicho de otra manera, el grado de diversificación internacional de las EMs no favorece (ni perjudica) que la puesta en marcha de iniciativas socialmente responsables con empleados, minorías sociales y comunidad local mejoren (empeoren) su marcha financiera.

Sin embargo, una alta diversificación internacional por parte de las EMs sí contribuye positivamente a que las prácticas de RSC vinculadas a los comportamientos medioambientales e impacto de los productos (Factor 2) redunden en una mejora sustancial del desempeño financiero. Por tanto, la diversificación internacional ejerce un efecto moderador positivo en la relación existente entre las iniciativas de RSC englobadas en el Factor 2 y el desempeño financiero (Figura 1). Por tanto, los resultados muestran un apoyo parcial a la Hipótesis 2.

El apoyo parcial de la Hipótesis 2 puede estar motivado por el distinto grado de visibilidad de las prácticas de RSC llevadas a cabo por las EMs (Chiu y Sharfman, 2011). Prácticas de RSC altamente visibles mejoran la legitimidad de las empresas y otorgando licencia para operar en diferentes mercados. Los aspectos medioambientales y los derivados del propio producto son más visibles por los grupos de interés que las iniciativas vinculadas con las relaciones con la comunidad local, empleados y protección de minorías sociales. 


\section{Figura 1}

\section{Efecto moderador de la diversificación internacional de las EMs en la relación RSC (Factor 2) - Resultado financiero}

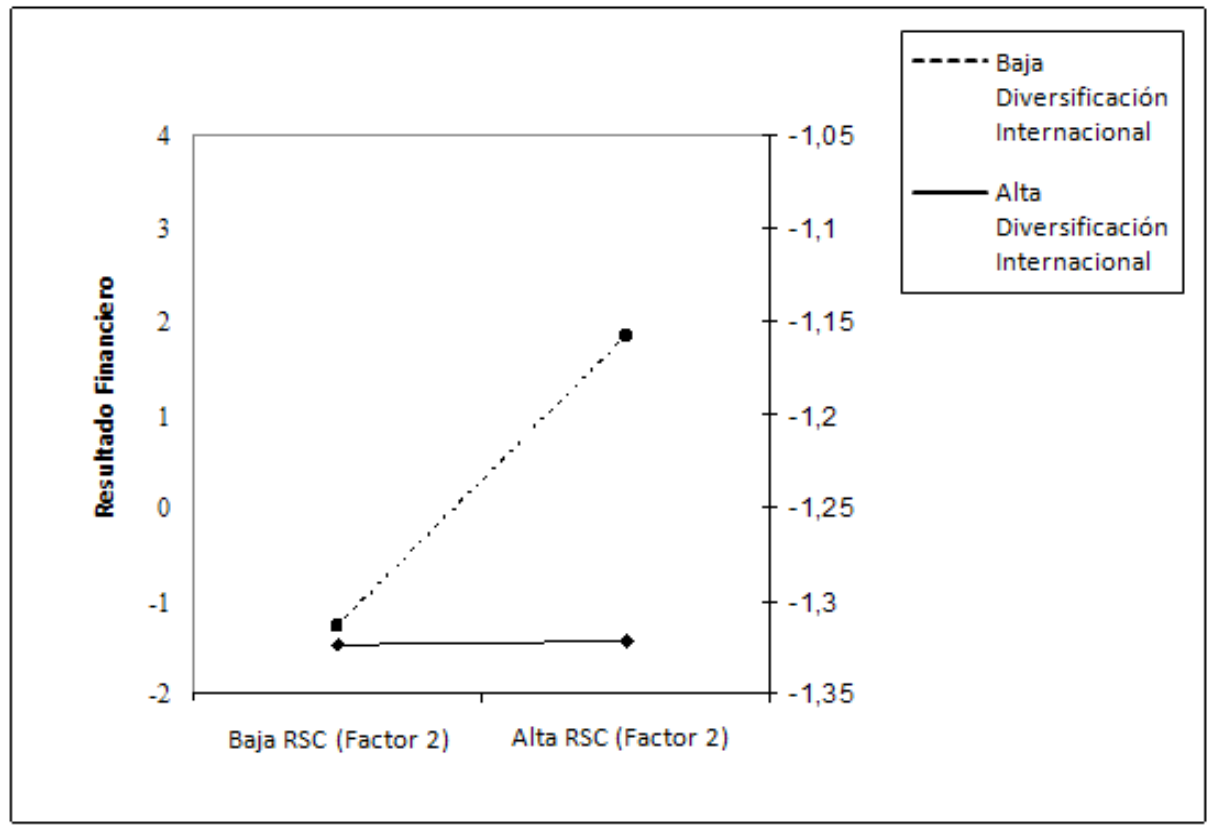

Fuente: Elaboración propia, a partir del resultado financiero utilizando datos de Standard \& Poors' (Capital IQ) y de los resultados obtenidos del análisis de regresión.

Los aspectos relacionados con los productos tienen también una elevada visibilidad porque los consumidores finales pueden captar directamente, a través de su compara y uso, sus beneficios sociales. Por ejemplo, productos con bajo impacto medioambiental $\mathrm{o}$ que contribuyan a la mejora de la salud y bienestar. Además, existen numerosas iniciativas medioambientales como el uso de materias primas y energías renovables, selección de suministradores sensibles por el medioambiente, mejoras del procedimiento productivo, uso del reciclaje y gestión de residuos, que pueden ser trasladadas a la sociedad mediante una adecuada estrategia de marketing, ganando así visibilidad.

\section{Conclusiones}

Las EMs juegan un papel esencial a la hora de llevar a cabo iniciativas locales y globales en de responsabilidad social, independientemente de su actividad principal. Dichas iniciativas pueden ser muy variadas y estar relacionadas con la problemática medioambiental, el fomento del bie-nestar en áreas vinculadas con la protección de derechos humanos, la educación, la promoción del empleo y la riqueza regional, o la inversión en investigación y desarrollo.

Sin embargo, la puesta en marcha de iniciativas socialmente responsables no implica necesariamente una mejora en el resultado financiero de las EMs. Para 
mejorar la comprensión de la relación entre el comportamiento social de las empresas $y$ el rendimiento financiero, diversos autores ponen de relieve la importancia de considerar factores que pueden influir en la relación entre ambas variables.

Siguiendo esta línea de investigación, este trabajo profundiza en el análisis de la relación entre RSC y desempeño financiero incorporando el efecto moderador de la diversificación internacional de las EMs y se apoya en la teoría del conocimiento. Las EMs adquieren conocimiento diverso de los diferentes mercados en los que operan. Gracias a este conocimiento las EMs pueden desarrollar iniciativas visibles de RSC con repercusión positiva en su desempeño financiero.

La diversificación internacional de las EMs constituye un factor clave para que las iniciativas de RSC encaminadas a proteger el medio ambiente así como las relacionadas con la puesta a disposición de productos innovadores que contribuyan al bienestar social sean valoradas por los grupos de interés, redundando en una mejora sustancial del desempeño financiero. Por el contrario, la diversificación internacional de las EMs no va a ayudar a rentabilizar las iniciativas sociales encaminadas a satisfacer los intereses y necesidades de la comunidad local, los empleados y de los colectivos sociales minoritarios.

Este trabajo tiene también
importantes implicaciones para los directivos y los poderes públicos. Los directivos deben incorporar en la estrategia de la empresa aspectos de RSC, considerando dichas iniciativas útiles y beneficiosas no sólo para la sociedad en su conjunto, sino también para la propia empresa al ser fuente de ventaja competitiva. Este esfuerzo debe ser más evidente en el caso de empresas que operan en varios países puesto porque deben satisfacer las demandas e intereses de diferentes colectivos sociales.

Además, los directivos de las empresas no sólo deben prestar interés a las iniciativas sociales más visibles por los grupos de interés, sino también a aquellas prácticas más difíciles de captar por la sociedad en su conjunto. Para incrementar la visibilidad de las prácticas de RSC se recomienda adoptar medidas encaminadas a dar a conocer las acciones de la empresa en materia social, tales como campañas de publicidad y promoción con los entes locales, o publicar los códigos de conducta con los empleados de la empresa o con grupos más desfavorecidos.

En cuanto a los poderes públicos y reguladores, estos deben crear políticas y legislaciones que favorezcan la adopción de iniciativas sociales por parte de las EMs. Para ello, deben destinar esfuerzos para homogeneizar las reglas de juego en los diferentes mercados con el fin de evitar comportamientos oportunistas por parte de las empresas. Dichos esfuerzos pueden materializarse por medio de incentivos económicos a organizaciones socialmente responsables así como fomentando colaboraciones con otras instituciones en materia social y medioambiental.

En definitiva, las instituciones públicas deben ayudar a que las EMs lleven a cabo una estrategia transnacional de sus prácticas de RSC contemplando las necesidades y demandas de cada entorno local así como fortaleciendo los estándares sociales y medioambientales propios a lo largo de su entramado organizativo con el fin de generar economías de escala y ventajas competitivas.

Finalmente, se señalan las limitaciones del presente trabajo $y$ 
Responsabilidad social y desempeño financiero en multinacionales...

Aguilera-Caracuel, Javier; Guerrero-Villegas, Jaime y Morales-Raya, Matilde

las futuras líneas de investigación. En primer lugar, se analiza la diversificación internacional agrupando países institucionalmente similares en la misma región. La agrupación de países en regiones recibe apoyo por parte de literatura previa (Yeoh, 2004). No obstante, al realizar esta agrupación se podría perder información relevante acerca de la existencia de peculiaridades culturales e institucionales propias de ciertos países.

En segundo lugar, en futuros trabajos de investigación resultaría muy interesante contrastar los resultados con una muestra de EMs de diferentes sectores de actividad con matrices ubicadas en diferentes países. Adicionalmente, cabe tener en cuenta el papel a desempeñar por la actuación de la empresa en países en vías de desarrollo en las citadas relaciones objeto de este estudio.

De hecho, las empresas que operan en países subdesarrollados deben decidir si se aprovechan de las circunstancias desfavorables de esos entornos en su propio beneficio en el corto plazo (por ejemplo, deslocalizando algunas de sus actividades a países en los que la protección legal en materia laboral o medioambiental es menos avanzada). O bien, dichas empresas deben decidir si destinan esfuerzos para mejorar el grado de bienestar social del lugar en el que operan, fomentando así la riqueza económica de la zona. Esta última postura puede acarrear beneficios muy positivos para la empresa a largo plazo ligados al aumento de la legitimidad, la transparencia y la coherencia interna de las EMs.

\section{Referencias bibliográficas}

Aguilera-Caracuel, Javier; Hurtado-Torres, Nuria E. y Aragón-Correa, Juan
A. (2012), Does international experience help firms to be green? A knowledge-based view of how international experience and organisational learning influence proactive environmental strategies. International Business Review, Vol. 2, № 5 , pp. 847-861.

Andersson, Svante. y Wictor, Ingemar (2003), Innovative internationalization in new firms: Born-globals - the Swedish case. Journal of International Entrepreneurship, Vol. 1, $\mathrm{N}^{\circ} 3$, pp. 249-267.

Aragón-Correa, Juan A. y Sharma, Sanjay (2003), A contingent resourcebased view of proactive corporate environmental strategy. Academy of Management Review, Vol. 28, $\mathrm{N}^{\circ} 1$, pp. 71-88.

Autio, Erkko; Sapienza, Harry J. y Almeida, James G. (2000), Effects of age at entry, knowledge intensity, and imitability on international growth. Academy of Management Journal, Vol. 43, N 5, pp. 909-924.

Bansal, Pratima (2005), Evolving sustainably: A longitudinal study of corporate sustainable development. Strategic Management Journal, Vol. 26, $\mathrm{N}^{\circ} 3$, pp. 197-218.

Barkema, Harry.G. y Vermeulen, Freek (1998), International expansion through start-up or acquisition: A learning perspective. Academy of Management Journal, Vol. 41, $\mathrm{N}^{0} 1$, pp. 7-26.

Barnett, Michael L. (2007), Stakeholder influence capacity and the variability of financial returns to corporate social responsibility. Academy of Management Review, Vol. 32, $\mathrm{N}^{\circ} 3$, pp. 794-816.

Barney, Jay (1991), Firm resources and sustained competitive advantage. Journal of Management. Vol. 17, № 1, pp. 99-121.

Bartlett, Christopher A. y Ghoshal, Sumantra (1989), Managing across borders: The transnational solution. Boston: Harvard Business School Press.

Bauer, Rob; Derwall, Jeroen y Otten, Rogér (2007), The ethical mutual fund 
performance debate: New evidence from Canada. Journal of Business Ethics, Vol. 70, № 2, pp. 111-124.

Blomstermo, A.; Eriksson, K. y Sharma, D. (2004), Domestic activity and knowledge development in the internationalization process of firms. Journal of International Entrepreneurship. Vol. 2, $\mathrm{N}^{\circ} 3$, pp. 239-259.

Casillas, José C.; Acedo, Francisco J. y Barbero, José L. (2010), Learning, unlearning and internationalisation: Evidence from the pre-export phase. International Journal of Information Management. Vol. 30, № 2, pp. 162 173.

Caves, Richard E. (1996), Multinational Enterprise and Economic Analysis. Segunda edición. Cambridge University Press: Cambridge.

Chetty, Sylvie; Eriksson, Kent y Lindbergh, J. (2006), The effect of specificity of experience on a firm's perceived importance of institutional knowledge in an ongoing business. Journal of International Business Studies. Vol. 37, N 5/6, pp. 699-712.

Chih, Hsiang-Lin; Chih, Hsiang-Hsuan y Chen, Tzu-Yin (2010), On the determinants of corporate social responsibility: International evidence on the financial industry. Journal of Business Ethics, Vol. 93, pp. 115-135.

Chiu, Shih-chi y Sharfman, Mark (2011), Legitimacy, Visibility, and the Antecedents of Corporate Social Performance: An Investigation of the Instrumental Perspective. Journal of Management, Vol. 37, $\mathrm{N}^{\circ} 6, \mathrm{pp}$. 1558-1585.

Choi, Jaepil y Chang, Heli (2009), Stakeholder relations and the persistence of corporate financial performance. Strategic Management Journal. Vol. 30, № 8, pp. 895-907.

Christmann, Petra (2004), Multinational companies and the natural environment: Determinants of global environmental policy standardization. Academy of Management Journal, Vol. 47, N 5 , pp. 747-760.

Christmann, Petra y Taylor, Glen (2001),
Globalization and the environment: Determinants of firm self-regulation in China. Journal of International Business Studies, Vol. 32, $\mathrm{N}^{\circ} 3$, pp. 439-458.

Cragg, Wesley (2005), Ethics, codes, corporations and the challenge of globalization, London: Edward Elgar Publishing.

Deephouse, David L. (2000), Media reputation as a strategic resource: an integration of mass communication and resource-based theories. Journal of Management, Vol. 26, $\mathrm{N}^{\circ} 6, \mathrm{pp}$ 1091-1112.

Durán-Herrera, Juan J. y Bajo-Davó, Nuria (2013), Incidencia de las variables institucionales del país de origen de las empresas multinacionales en su performance financiero. Universia Business Review, primer trimestre, pp. 52-67.

Eriksson, Kent; Johanson, Jan; Majkgard, Anders. y Sharma, Deo. D. (2000), Effect of variation on knowledge accumulation in the internationalization process. International Studies of Management and Organization, Vol. $30, N^{\circ} 1$, pp. 26-45.

Fombrun, Charles (1996), Reputation: Realizing Value from the Corporate Image, Harvard Business School Press: Boston, MA.

Freeman, R. Edward (1984), Strategic Management: A stakeholder approach. Pitman Press, Boston,

Friedman, Milton (1970), The social responsibility of business is to increase its profits. New York Times Magazine (reprinted from Perspectives in Business Ethics, 3rd ed., 280-285, by Hartman, L.P., Ed. 2005, New York, NY: McGraw-Hill).

Gómez-García, Francisco (2008), Responsabilidad social corporativa y performance financiero: Treinta y cinco años de investigación empírica en busca de un consenso. Principios: Estudios de Economía Política, Vol. 11, pp. 5-24.

Grant, Robert M. (1987), Multinationality and performance among British manufacturing companies. Journal 
Responsabilidad social y desempeño financiero en multinacionales...

Aguilera-Caracuel, Javier; Guerrero-Villegas, Jaime y Morales-Raya, Matilde

of International Business Studies, Vol. 18, № 3, pp. 79-90.

Hair, Joseph F.; Anderson, Rolph E.; Tatham Ronald L. y Black, William C. (2009). Multivariate Analysis. Sexta edición. Madrid: Prentice Hall.

Han, Ki C.; Lee, Suk Hun y Suk, David Y. (1998), Multinationality and firm performance. Multinational Business Review, Vol. 6, $\mathrm{N}^{\circ} 2$, pp. 63-70.

Hart, Stuart L. (1995), A natural resourcebased view of the firm. Academy of Management Review, Vol. 20, $\mathrm{N}^{\circ} 4$ pp. 986-1014.

Hausman, Jerry (1978), Specification test in economic. Econometrica, Vol. 46, $\mathrm{N}^{\circ}$ 6, pp. 1251-1271.

Hitt, Michael A.; Hoskisson, Robert E. y Kim, Hicheon (1997), International diversification: Effects on innovation and firm performance in productdiversified firms. Academy of Management Journal, Vol. 40, $\mathrm{N}^{\circ} 4$, pp. 767-799.

Hitt, Michael A.; Tihanyi, Laszlo; Miller, Toyah y Connelly, Brian (2006), International diversification: Antecedents, outcomes, and moderators. Journal of Management, Vol. 32, $\mathrm{N}^{\circ} 6$, pp. 831-867.

Hoffman, Andrew J. (2000), Competitive environmental strategy: A guide to the changing business landscape. Washington, DC: Island Press.

Howard-Grenville, Jennifer A. y Hoffman, Andrew J. (2003), The importance of cultural framing to the success of social initiatives in business. Academy of Management Executive, Vol. 17, № 2, pp. 70-84.

Hull, Clyde E. y Rothenberg, Sandra (2008), Firm performance: The interactions of corporate social performance with innovation and industry differentiation. Strategic Management Journal, Vol. $29, N^{\circ} 7$, pp. 781-789.

Hymer, Stephen H. (1976), The international operations of national firms: A study of foreign direct investment. Cambridge, MA: MIT press.

Jensen, Michael C. (2001), Value maximization, stakeholder theory, and the corporate objective function. Business Ethics Quarterly, Vol. 12, $\mathrm{N}^{\circ} 2$, pp. 235-256.

Johanson, Jan y Vahlne, Jan-Erik (1977), The internationalization process of the firm. A model of knowledge development and increasing foreign market commitments. Journal of International Business Studies, Vol. 8, № 1, pp. 23-32.

Karim, Khondkar E.; Pinsker, Robert y Robin, Ashok (2013), Firm size and the voluntary disclosure of nonfinancial information by private versus public firm managers. Managerial Auditing Journal, Vol. 28, № 9, pp. 866-892.

Kaufmann, Lutz y Körte, Peter (2010), Responses of Advanced Country MNEs to Low-cost Country Imports in Their Home Markets. Management International Review, Vol. 50, pp. 241-262

King, Andrew A. y Shaver, J. Miles (2001). Are aliens green? Assessing foreign establishments' environmental conduct in the United States. Strategic Management Journal, Vol. 22, № 11, pp. 1069-1086.

Kogut, Bruce (1985), Designing global strategies: Profiting from operational flexibility. Sloan Management Review, Vol. 27, № 1, pp. 27-38.

Kolk, Ans y Pinkse, Jonatan (2008), A perspective on multinational enterprises and climate change: Learning from "an inconvenient truth. Journal of International Business Studies, Vol. 39 , № 8 , pp. 13591378

Kolk, Ans y van Tulder, Rob (2010), International business, corporate social responsibility and sustainable development. International Business Review, Vol. 39, № 8, pp. 1359-1378.

Kostova, Tatiana y Roth, Kendall (2002), Adoption of an organizational practice by subsidiaries of multinational corporations: Institutional and relational effects. Academy of Management Journal, Vol. 45, № 1 , pp. 215-233. 
Kostova, Tatiana; Roth, Kendall y Dacin, Tina (2008), Institutional theory in the study of multinational corporations: A critique and new directions. Academy of Management Review, Vol. 33, No 4, pp. 994-1006.

Kotabe, Masaaki (1990), The relationship between offshore sourcing and innovativeness of US multinational firms: An empirical investigation. Journal of International Business Studies, Vol. 21, № 4, pp. 623-639.

Lichtenstein, Donald R.; Drumwright, Minette E. y Braig, Bridgette M. (2004), The effects of corporate social responsibility on customer donations to corporate supported nonprofits. Journal of Marketing, Vol. $68, \mathrm{~N}^{\circ} 4$, pp. 16-32.

Luo, Yadong (2001), Determinants of entry in an emerging economy: A multilevel approach. Journal of Management Studies, Vol. 38, № 3, pp. 443-472.

Marcus, Alfred y Fremeth, Adam (2009), Green management matters regardless. Academy of Management Perspectives, Vol. 23, № 3 , pp. 1726.

Margolis, Joshua D. y Walsh, James P. (2001), People and Profits? The Search for a Link between a Company's Social and Financial Performance. Mahwah, NJ: Lawrence Erlbaum.

Margolis, Joshua D. y Walsh, James P. (2003), Misery loves companies: rethinking social initiatives by business. Administrative Science Quarterly, Vol. 48, No 2, pp. 268-305.

McWilliams, Abagail y Siegel Donald (2000), Corporate social responsibility and financial performance: Correlation or misspecification?. Strategic Management Journal, Vol. 21, $\mathrm{N}^{\circ} 5$, pp. 603-609.

McWilliams, Abagail y Siegel, Donald (2001), Corporate social responsibility: a theory of the firm perspective. Academy of Management Review, Vol. 26, N ${ }^{\circ} 1$, pp. 117-127.

Nachum, Lilach. y Zaheer, Srilata (2005), The persistence of distance? The impact of technology on MNE motivations for foreign investment. Strategic
Management Journal, Vol. 26, № 8 , pp. 747-767.

Nonaka, Ikujiro (1994), A dynamic theory of organizational knowledge creation. Organization Science, Vol. 5, № 1 , pp. $14-29$.

Nunnally, Jum C. y Bernstein, Ira H. (1995), Teoría de la Psicometría. New York: McGraw Hill.

Orlitzky Marc; Schmidt, Frank L. y Rynes, Sara L. (2003), Corporate social and financial performance: A meta analysis. Organization Studies, Vol. 24, pp. 403-441.

Peng, Mike W.; Wang, Denis Y.L. y Jiang, Yi (2008), An institution-based view of international business strategy: A focus on emerging economies. Journal of International Business Studies, Vol. 39, № 5, pp. 920-936.

Perrini, Francesco; Russo, Angeloantonio; Tencati, Antonio y Vurro, Clodia (2011), Deconstructing the relationship between corporate social and financial performance. Journal of Business Ethics, Vol. 102, Supplement 1, pp. 59-76.

Petersen, Bent; Pedersen, Torben y Lyles, Marjorie A. (2008), Closing knowledge gaps in foreign markets. Journal of International Business Studies, Vol. 39, № 7, pp. 1097-1113.

Porter, Michael E. y van der Linde, Claas (1995), Green and competitive: Ending the stalemate. Harvard Business Review, Vol. 73, № 5, pp. 120-134.

Post, James E.; Preston, Lee E. y Sachs, Sybille (2002), Managing the extended enterprise: The new stakeholder view. California Management Review, Vol. 45, No 1, pp. 6-28.

Prahalad, C.K. y Hamel, Gary (1994), Strategy as field of study: Why search for a new paradigm? Strategic Management Journal, Vol. 15, № 2, pp. 5-16.

Ramus, Catherine A. y Steger, Ulrich (2000), The roles of supervisory support behaviors and environmental policy in employee 'ecoinitiatives' at leading-edge European companies. Academy of Management Journal Vol. 43, N 4, pp. 605-626. 
Razafindrambinina, Dominique y Sabran, Annisa (2014), The Impact of Strategic Corporate Social Responsibility on Operating Performance: An Investigation Using Data Envelopment Analysis in Indonesia. Journal of Business Studies Quarterly, Vol. 6, $\mathrm{N}^{\circ}$ 1, pp. 68-78.

Rugman, Alan M. (1981), Inside the multinationals: The economics of internal markets. New York: Columbia University Press.

Rugman, Alan M. y Verbeke, Alain (1998), Corporate strategies and environmental regulations: An organizing framework. Strategic Management Journal, Vol. 19, $\mathrm{N}^{\circ} 4$, pp. 363-375.

Russo, Michael V. y Fouts, Paul A. (1997), $A$ resource-based perspective on corporate environmental performance and profitability. Academy of Management Journal, Vol. 40, $\mathrm{N}^{\circ} 3$, pp. 534-559.

Russo, Michael V. y Harrison, Niran S. (2005), Organizational design and environmental performance: Clues from the electronic industry. Academy of Management Journal, Vol. 48, $\mathrm{N}^{\circ}$ 4, pp. 582-593.

Sambharya, Rakesh B. (1995), The combined effect of international diversification and product diversification strategies on the performance of US based multinational corporations. Management International Review, Vol. 35, № 3, pp. 197-218.

Scherer, Andreas G. y Palazzo, Guido (2011), The new political role of business in a globalized world: A review of a new perspective on CSR and its implications for the firm, governance, and democracy. Journal of Management Studies, Vol. 48, № 4, pp. 899-931.

Sharfman, Mark y Fernando, Chitru S. (2008), Environmental risk management and the cost of capital. Strategic Management Journal, Vol. 29, pp. 569-592.

Strike, Vanessa M.; Gao, Jijun y Bansal, Pratima (2006), Being good while being bad: Social responsibility and the international diversification of
US firms. Journal of International Business Studies, Vol. 37, № 6, pp. 850-862.

Strong, Kelly C.; Ringer, Richard C. y Taylor, Steven A. (2001), THE* ruled of stakeholder satisfaction (*timeliness, honesty, empathy). Journal of Business Ethics, Vol. 32, $\mathrm{N}^{\circ} 3$, pp. 219-230.

Sundaram, Anant K. y Inkpen, Andrew C. (2004), Stakeholder theory and "the corporate objective revisited": A reply. Organization Science, Vol. 15, $\mathrm{N}^{\circ} 3$, pp. 370-371.

Tallman Stephen y Li Jiatao (1996), Effects of international diversity and product diversity on the performance of multinational firms. Academy of Management Journal, Vol. 39, pp. 179-196.

Tencati, Antonio y Zsolnai, Laszlo (2009), The collaborative enterprise. Journal of Business Ethics, Vol. 85, № 3 , pp. 367-376.

Thomas, Douglas E. y Eden, Lorraine (2004), What is the shape of the multinationality-performance relationship? Multinational Business Review, Vol. 12, № 1, pp. 89-99.

Ullman, Arieh A. (1985), Data in search of a theory: A critical examination of the relationship among social performance, social disclosure and economic performance of US firms. Academy of Management Review, Vol. 10, N 3, pp. 540-557.

Van Raaij, W. Fred (1997), Globalization of marketing communication?. Journal of Economic Psychology, Vol. 18, No 2, pp. 259-270.

Venkatraman, N. (1989), The concept of fit in strategy research: Toward verbal and statistical correspondence. Academy of Management Review, Vol. 14, No 3, pp. 423-444.

Vogel, David J. (2005), Is there a market for virtue? The business case for corporate social responsibility. California Management Review, Vol. $47, N^{\circ} 4$, pp. 19-45.

Waddock, Sandra A. (2004), Parallel universes: Companies, academics, 
and the progress of corporate citizenship. Business and Society Review, Vol. 109, № 1, pp. 303-319.

Waddock, Sandra A. y Graves, Samuel B. (1997), The corporate social performance-financial performance link. Strategic Management Journal, Vol. 18, № 4, pp. 303-319.

Yang, Fu-Ju; Lin, Ching-Wen y Chang, YungNing (2010), The linkage between corporate social performance and corporate financial performance. African Journal of Business Management, Vol. 4, № 4, pp. 406413.
Yeoh, Poh-Lin (2004), International learning: Antecedents and performance applications among newly internationalizing companies in an exporting context. Journal of International Business Studies, Vol. 21, No $4 / 5$, pp. 511-535.

Zahra, Shaker A. (2005), A theory of international new ventures: A decade of research. Journal International Business Studies, Vol. 36, № 1 , pp. 20-28. 